\title{
Detecting poisoning of metal oxide gas sensors at an early stage by temperature cycled operation
}

\author{
Marco Schüler, Thomas Fricke, Tilman Sauerwald, Andreas Schütze \\ Laboratory for Measurement Technology, Saarland University, Saarbrücken, Germany \\ m.schueler@Imt.uni-saarland.de
}

\begin{abstract}
:
In this paper we study the effect of $\mathrm{HMDSO}$ (hexamethyldisiloxane) on an $\mathrm{SnO}_{2}$ metal oxide gas sensor in temperature cycled operation (TCO). Using TCO, the sensor discriminates between pure air, carbon monoxide and ethanol, both in the range of $1 \mathrm{ppm}$ to $5 \mathrm{ppm}$. We could show that gas identification is still possible after poisoning with 340 ppm*min HMDSO, i.e. a HMDSO poisoning dose that fulfills the requirement of DIN EN 50194-1. Moreover, the poisoning dose can be quantified with a resolution of $\pm 85 \mathrm{ppm}^{*} \mathrm{~min}$ again using data recorded in TCO mode, providing an easy method for early detection of HMDSO poisoning and a novel approach for sensor self-monitoring.
\end{abstract}

Key words: metal oxide gas sensor, temperature cycled operation, sensor self-monitoring, sensor poisoning, HMDSO vapor generation

\section{Introduction}

Metal oxide (MOX) gas sensors are available at relatively low cost and can detect a broad range of reducing and oxidizing gases with high sensitivity. They take advantage of resistance changes in the material, usually a granular metal oxide. Its resistance is influenced by the adsorption of oxygen, which creates a depletion layer at the interface between its grains. This depletion layer acts as an energy barrier and thus determines the resistance of the material $[1,2,3]$.

The interaction of adsorbed oxygen with reducing or oxidizing gases depends strongly on the temperature or, in the case of temperature cycled operation (TCO) $[4,5]$, the corresponding temperature profile.

In TCO, sensor temperature is varied in a defined manner, allowing the study of the sensor's dynamic resistance properties during fast temperature changes as well as at defined temperature levels. It has been shown that TCO can increase selectivity $[5,6,7]$, and can also further increase the sensitivity of MOX gas sensors [8]. In this paper, we examine if TCO can also be used for an early detection of sensor poisoning.

\section{Experimental setup and determination of HMDSO vapor pressure}

DIN EN 50194-1 [9] describes test methods for detectors of combustible gases in domestic premises. It requires such detectors to operate properly after being exposed, among others, to $10 \pm 3$ ppm HMDSO (hexamethyldisiloxane) over a time period of 40 minutes.

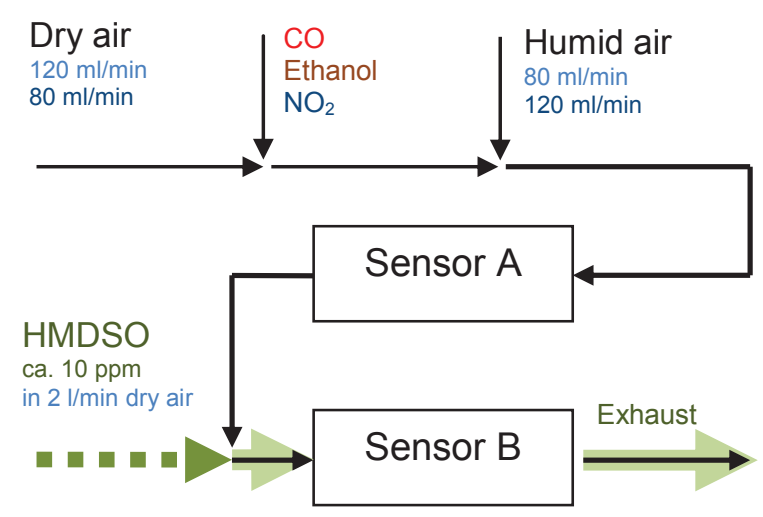

Fig. 1: gas mixing apparatus (GMA) setup for studying the influence of HMDSO poisoning

Fig. 1 shows the setup of the gas mixing apparatus for the study of the influence of defined HMDSO concentrations on TCO-based measurements. $\mathrm{CO}$, ethanol and $\mathrm{NO}_{2}$ were provided from test gas bottles. Gas flows were regulated with standard mass flow controllers.

Air humidification was realized in a glass bubbler at ambient temperature $\left(25^{\circ} \mathrm{C}\right)$. HMDSO was provided from a second glass bubbler, which is temperature-regulated by a thermostat (LAUDA RE 307). An air flow of $0.5 \mathrm{ml} / \mathrm{min}$ is saturated with HMDSO vapor in the temperature-regulated bubbler bottle. It is led to a second air stream $(2000 \mathrm{ml} / \mathrm{min})$ for dilution. 


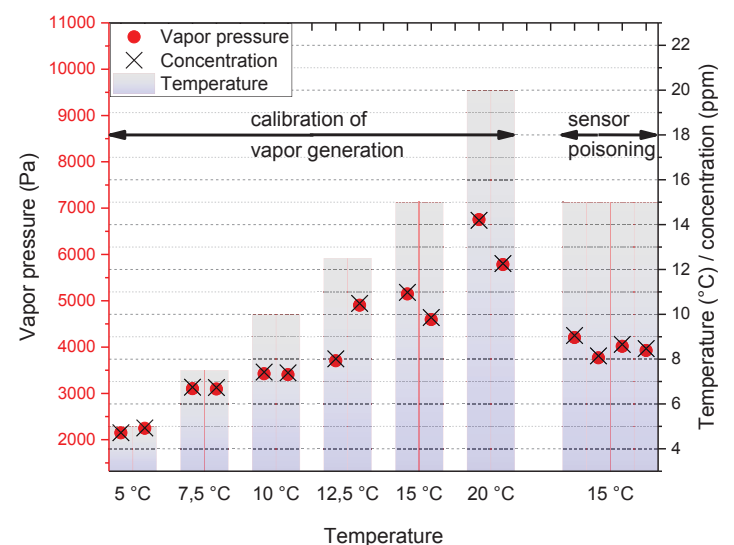

Fig. 2: Vapor pressures and resulting HMDSO concentrations at different temperatures. Left: preparatory measurements. Right: measurements during actual sensor poisoning

To achieve an HMDSO concentration in the range required by DIN EN 50194-1, we carried out periodic gravimetric measurements of the bubbler bottle over several hours, during which the bottle was flowed through by a constant air stream. The temperature of the water bath was varied from $5{ }^{\circ} \mathrm{C}$ up to $20^{\circ} \mathrm{C}$. The weight loss over periods of 21 to 56 hours was measured using a Precisa XT $1220 \mathrm{M}$ high precision electronic balance. Fig. 2 shows the corresponding vapor pressure and concentration values, which were calculated using the ideal gas law assuming an ambient pressure of $1013.25 \mathrm{hPa}$.
The left hand side of Fig. 2 shows the measurements carried out beforehand to determine which HMDSO bubbler temperature was suitable for the sensor poisoning measurement. At $15{ }^{\circ} \mathrm{C}$, the weight loss measured in the preparatory measurements results in a concentration of approx. $10 \mathrm{ppm}$ in the gas stream across the sensor.

During the gas measurements, we carried out further gravimetric measurements of the test gas bottle. The resulting concentrations were between 8 and $9 \mathrm{ppm}$, well within the range required by the DIN standard ( $10 \pm 3 \mathrm{ppm})$.

Fig. 3 shows the gas profile which is applied to sensor B. During the gas profile, the sensor is exposed to $1 \mathrm{ppm} / 5 \mathrm{ppm}$ of $\mathrm{CO}$ and ethanol, as well as $0.4 \mathrm{ppm} / 2 \mathrm{ppm}$ of NO2, each at $40 \%$ rh as well as at $60 \%$ rh. Every gas exposure lasts for $20 \mathrm{~min}$, followed by $20 \mathrm{~min}$ in pure humid air. The gas profile is repeated five times after an initial 12 hour run-in period at $40 \%$ rh. Sensor $A$ is exposed to the test gases mostly in the same manner as sensor $\mathrm{B}$, only differing during the 10 minutes period in which Sensor B is exposed to HMDSO: In this time period, sensor A remains exposed to pure air with $40 \%$ rh at a flow of $200 \mathrm{ml} / \mathrm{min}$, while sensor B is poisoned with approx. $8.5 \mathrm{ppm}$ HMDSO at $3 \%$ rh and an overall air flow of $2200 \mathrm{ml} / \mathrm{min}$.

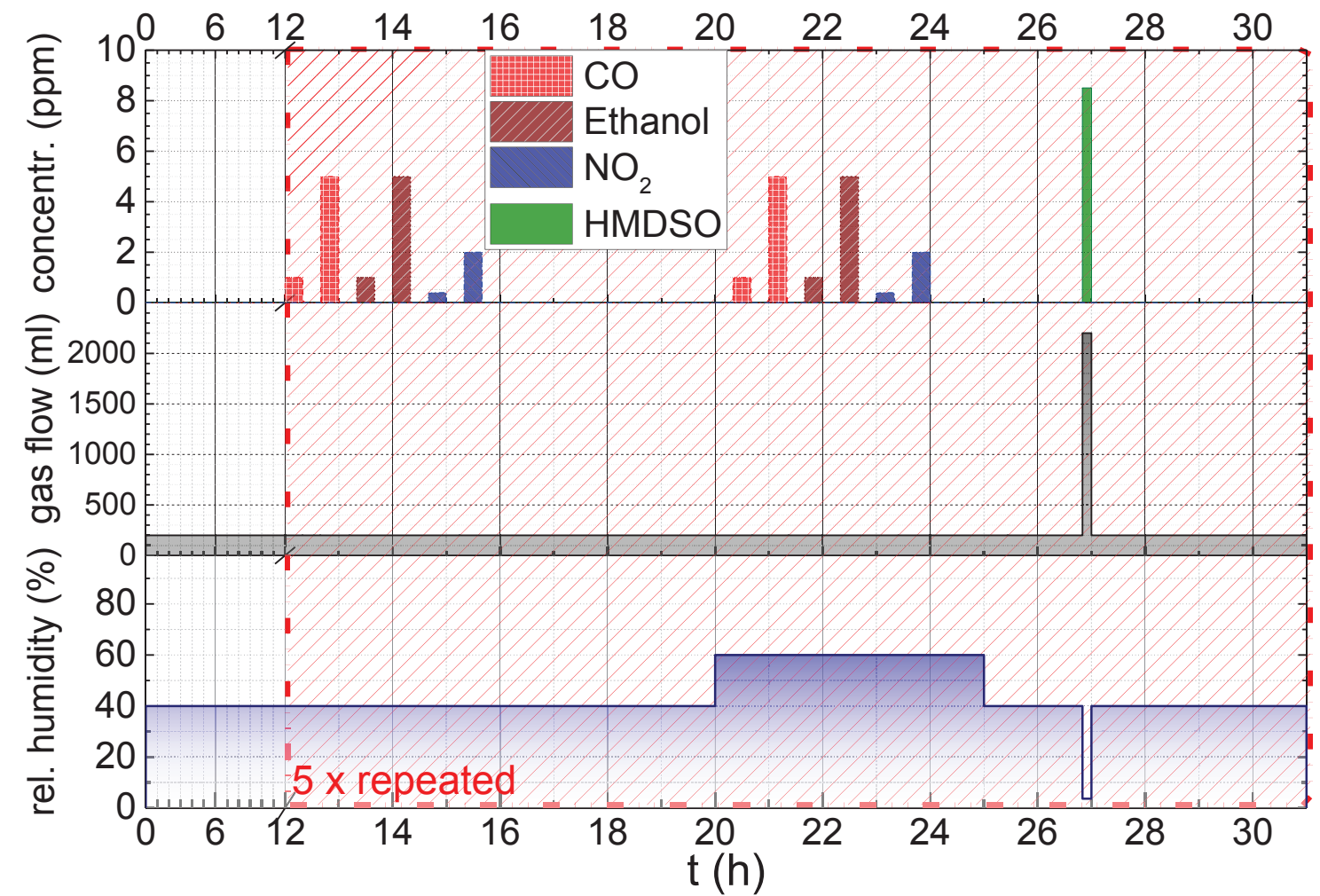

Fig. 3: Gas profile for test gas and HMDSO concentrations, overall gas flow and rel. humidity at sensor $B$. The section between $12 \mathrm{~h}$ and $31 \mathrm{~h}$ was repeated five times to study the increasing poisoning effect of HMDSO. 


\section{Sensor operation and feature extraction}

We examined a commercial $\mathrm{SnO}_{2}$-based broad range metal oxide gas sensor (GGS 1330, UST Umweltsensortechnik $\mathrm{GmbH}$, Geraberg). Both sensors were operated using the temperature cycle shown in the lower row of Fig. 4. The sensor temperature was controlled using the resistance of the integrated $\mathrm{Pt}$ heater. The conductance was measured every $20 \mathrm{msec}$ at a constant voltage of $250 \mathrm{mV}$. Sensor control and data acquisition was performed with the 3SToolbox (3S GmbH, Saarbrücken) sensor operation hardware for UST GGS sensors.

The data acquired during the $\mathrm{NO}_{2}$ exposures showed significant inconsistencies, probably caused by carryover effects due to the small concentrations and short exposures. The following evaluation is therefore confined to data recorded during exposure to pure humid air, $\mathrm{CO}$ and ethanol only.

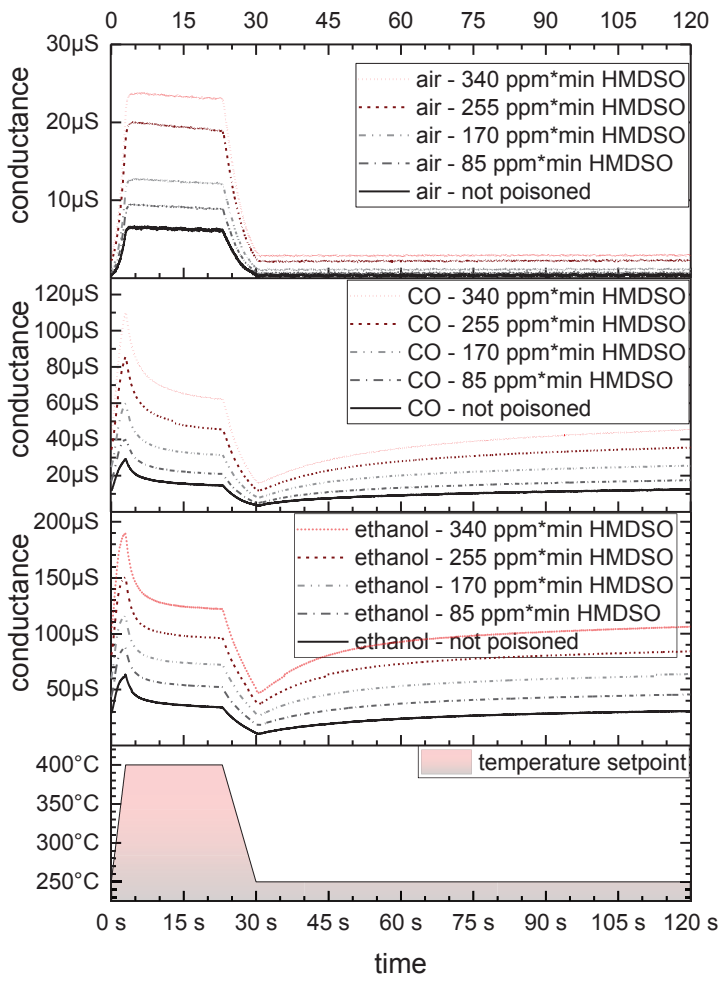

Fig. 4: Conductance values of sensor $B$ over time at $40 \%$ rh at different poisoning states. First row: conductance in pure air, second row: in air with $5 \mathrm{ppm} \mathrm{CO}$, third row: in air with $5 \mathrm{ppm}$ ethanol. Lower row: temperature cycle.

The upper rows of Fig. 4 show conductance values of sensor $\mathrm{B}$ over the temperature cycle in pure air and in the presence of $5 \mathrm{ppm} \mathrm{CO}$, and $5 \mathrm{ppm}$ ethanol, respectively, all at $40 \% \mathrm{rh}$, for the five repetitions of the gas profile, i.e. after different cumulative HMDSO doses. It is obvious from the graphs, that the exposure to HMDSO has a strong influence on the absolute conductance, while the shape of the conductance profile remains similar. This indicates that a multivariate analysis of the conductance profile, taking its shape into account, should be more stable with regard to poisoning than a monodimensional evaluation of the sensor conductance, i.e. the evaluation of only one point of the temperature cycle or operation at a constant temperature. A monodimensional evaluation would clearly carry the risk of false alarms after poisoning, as poisoning with 340 ppm*min HMDSO leads to a similar increase in conductance at the typical operating temperature of $400{ }^{\circ} \mathrm{C}$ as an exposure of the unpoisoned sensor to $5 \mathrm{ppm}$ CO.

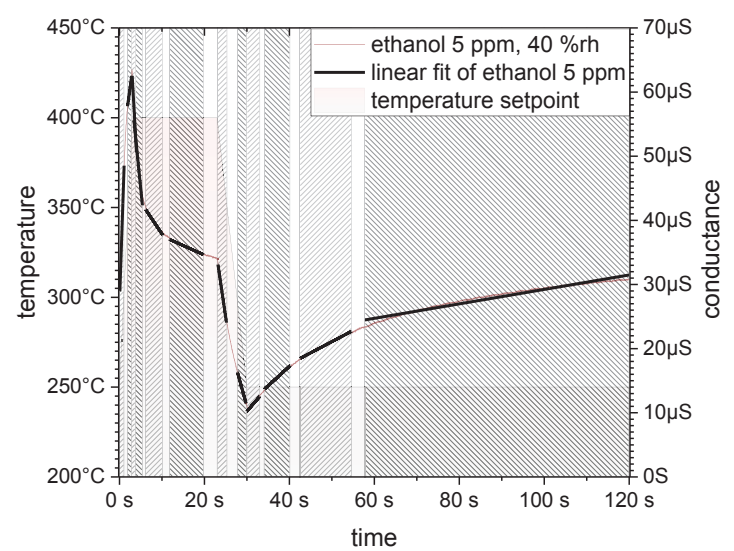

Fig. 5: Temperature cycle and conductance over time at an exemplary gas exposure the black lines represent the linear fits carried out in the shaded sections for feature extraction.

To enable multivariate analysis of the sensor data, it is necessary to represent the data in a reduced dataset. Fig. 5 shows an exemplary conductance profile and linear fits that were calculated for twelve separate sections of the temperature cycle. These linear fits yield a mean value and a slope for each section; they represent the conductance over time in a dataset (feature vector) of 24 numerical values per cycle, which is used for further analysis.

\section{Data analysis and results}

For further analysis of the measurement results, we used LDA (Linear Discriminant Analysis), a supervised algorithm for dimensionality reduction. It calculates a linear transformation projecting the feature vector into an $\mathrm{N}-1$ dimensional space, with $\mathrm{N}$ being the number of classes which are to be discriminated [10].

Fig. 6 exemplarily shows an LDA plot calculated from data sets of sensor $\mathrm{A}$, featuring all $\mathrm{CO}$ and ethanol exposures, as well as the corresponding air exposures at $40 \% \mathrm{rh}$ and $60 \% \mathrm{rh}$, respectively. It shows a good discrimination of both gases and air independent of relative 
humidity and varying gas concentrations contained in the data. This result indicates that ageing-induced drift has no significant influence on the separability of the different gases, at least for the test duration of 106 hours.

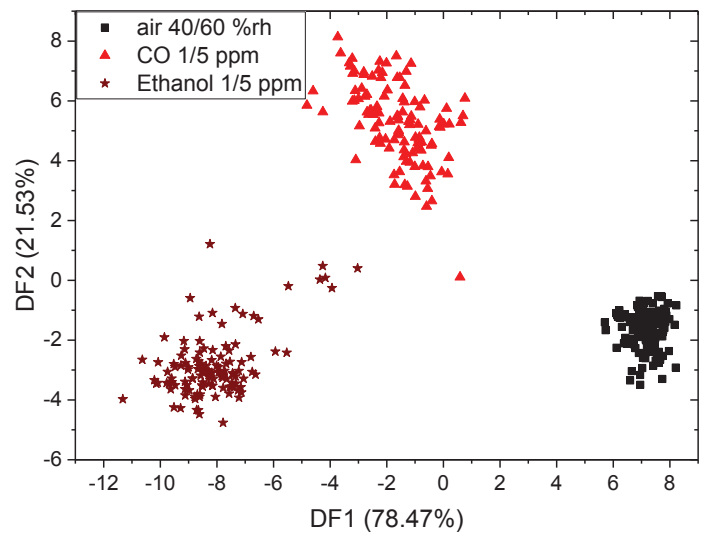

Fig. 6: Discrimination of all air, $\mathrm{CO}$ and ethanol exposures by sensor $A$, using $L D A$

Fig. 7 shows the corresponding LDA plot calculated from data of sensor $B$. The dataset contains data from different sensor states, corresponding to different HMDSO exposures. While the gas exposures are still fairly well separated from air (thus allowing reliable operation as long as there are only modest requirements on selectivity), the different classes are closer together and some outliers of the ethanol class are intersecting the $\mathrm{CO}$ group.

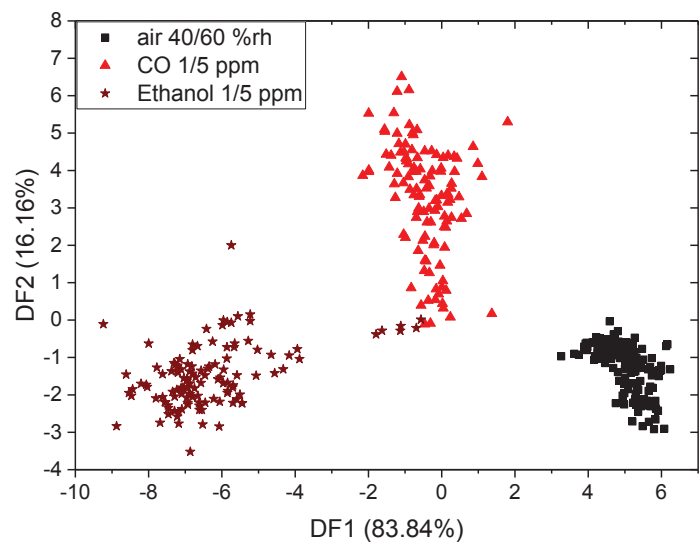

Fig. 7: Discrimination of all air, $\mathrm{CO}$ and ethanol exposures by sensor $B$, using $L D A$

Comparison with fig. 6 shows that there is a negative influence of HMDSO on the sensor performance, i.e. the selectivity in TCO mode. It is therefore desirable to detect the degradation of the sensor as early as possible, in order to allow replacement before its performance is no longer sufficient for the particular application.

A first approach to this goal is shown in fig. 8. We used the same data as in fig. 7 (sensor B with all $\mathrm{CO}$ and ethanol exposures and rh values). This time, five classes representing the poisoning state, i.e. the cumulative HMDSO exposure, were defined. While a rough estimation of the poisoning state seems possible, there is a significant overlap between classes. $A$ validation using the $k$-nearest neighbors ( $k$ NN) classifier with $k=3$ and the Euclidian distance yields a correct classification rate of $81.2 \%$; all misclassified points are attributed to a neighboring class, i.e. the poisoning state is sometimes over- or underestimated slightly.

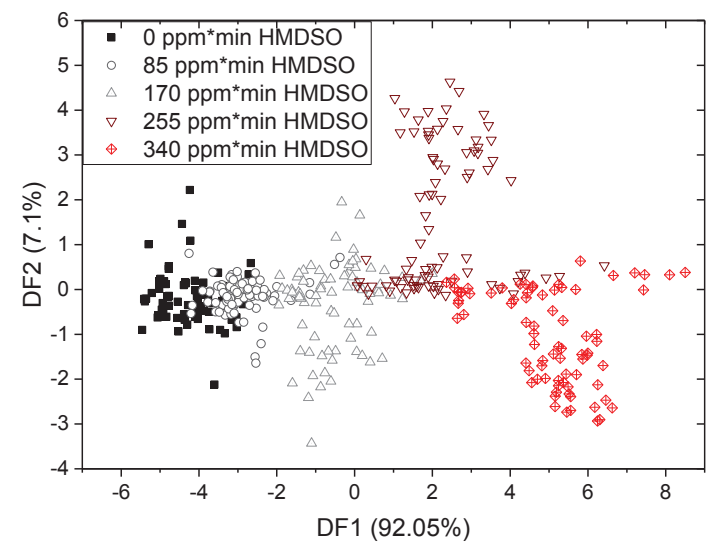

Fig. 8: Discrimination of five poisoning states of sensor B, using data from air, $\mathrm{CO}$ and ethanol exposures

When reducing the number of poisoning states, an improved classification, independent of the gas exposure, is achieved. Fig. 9 shows an LDA plot which is based on data from air, CO, and ethanol at three poisoning states $(0 / 170 /$ $\left.340 \mathrm{ppm}{ }^{*} \mathrm{~min}\right)$ only. The three different states are well separated from each other and can be classified correctly using the k-NN algorithm.

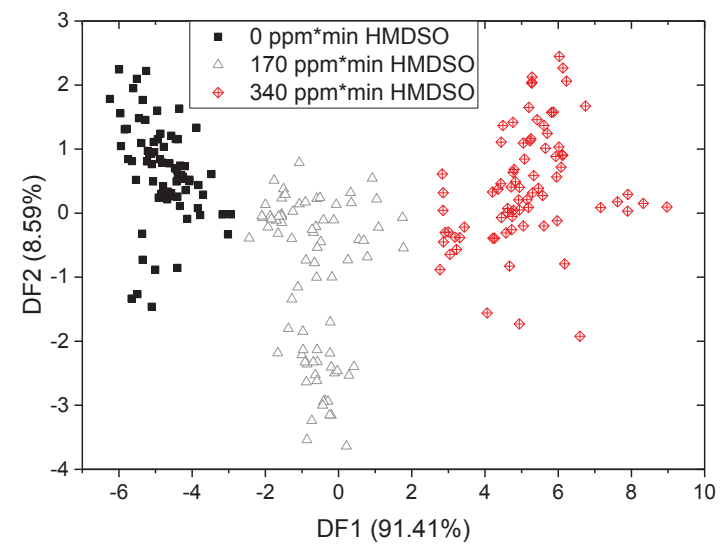

Fig. 9: Discrimination of three poisoning states of sensor B, using data from air, $\mathrm{CO}$ and ethanol exposures

For a more precise estimation of the sensor state, we propose a two-step approach: In a first step, we can determine if the sensor is operating in clean air or if a reducing gas is present. Fig. 7 showed that this is possible 
regardless of the poisoning state. If the sensor is operated in air, the poisoning state can be determined more precisely as shown in Fig. 10.

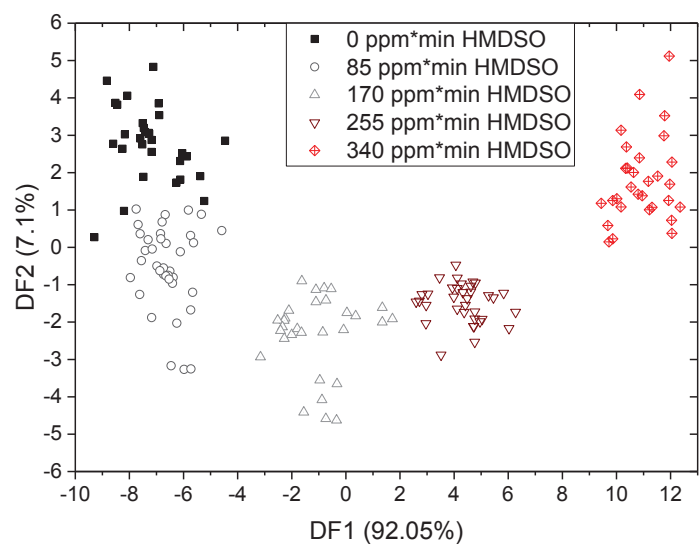

Fig. 10: Discrimination of five poisoning states of sensor $B$, using data from air exposures only

Fig. 10 shows an LDA calculated using data acquired only in air (both 40 and $60 \%$ rh) at five poisoning states. The classes overlap slightly, but $97.5 \%$ are classified correctly compared to only $81.2 \%$ in the one-step approach.

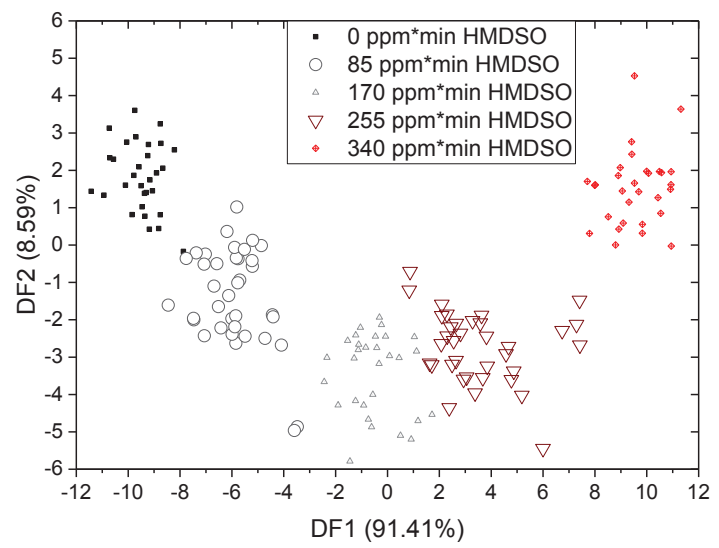

Fig. 11: LDA calculated with data acquired in air after 0/170/340 ppm*min cumulated HMDSO exposure, and applied to data acquired after $85 / 255 \mathrm{ppm}^{*} \mathrm{~min}$

Fig. 11 shows the same data, but the calculation of the LDA was carried out using data from three states only, i.e. 0/170/340 ppm*min HMDSO. Evaluation was carried out with data from all poisoning states. The fact that the intermediate poisoning states used only for evaluation are projected between the adjacent poisoning states used for calculating the LDA shows the stability of the approach.

\section{Conclusion and Outlook}

The results show that additionally to its benefits for selectivity and sensitivity, TCO enables a stable gas determination even after poisoning with HMDSO. Furthermore, it enables moni- toring the sensor's poisoning state, allowing quantification of the HMDSO exposure dose with an accuracy of $\pm 85 \mathrm{ppm}^{*} \mathrm{~min}$.

For this paper, only one sensor was poisoned by small HMDSO exposures, in a range which still allows proper sensor operation. In our ongoing work, we will study the possibility to transfer the results to a second sensor of the same type after a corresponding poisoning, as well as the influence of higher HMDSO exposures and other sensor impairments.

\section{References}

[1] Tricoli, A.; Righettoni, M.; and Teleki, A.: „Semiconductor gas sensors: dry synthesis and application." Angew. Chem. Int. Ed., 49(42): pp. 7632-59. doi: 10.1002/anie.200903801, 2010.

[2] Morrison, S. R.: "Semiconductor gas sensors", Sensors \& Actuators, 2, pp. 329-341, doi: 10.1016/0250-6874(81)80054-6, 1982.

[3] Kohl, D.: "Surface processes in the detection of reducing gases with $\mathrm{SnO}_{2}$-based devices", Sensors \& Actuators, 18, pp. 71-113, doi: 10.1016/0250-6874(89)87026-X, 1989.

[4] Lee, A.P.; and Reedy, B.J.: Temperature modulation in semiconductor gas sensing, Sensors \& Actuators B, 60, pp. 35-42, doi: 10.1016/S0925-4005(99)00241-5, 1999.

[5] Meier D.C.; Evju J.K.; Boger, Z.; Raman, B.; Benkstein, K.D., Martinez, C.J.; Montgomery, C.B.; and Semancik, S.: The potential for and challenges of detecting chemical hazards with temperature-programmed microsensors, Sensors \& Actuators B, 121, pp. 282-294, doi:10.1016/j.snb.2006.09.050, 2007.

[6] Schütze, A.; Gramm, A.; and Rühl, T.: „Identification of Organic Solvents by a Virtual Multisensor System with Hierarchical Classification“, IEEE Sensors Vol. 4, (6), pp. 857863, doi: 10.1109/JSEN.2004.833514, 2004.

[7] Leidinger, M.; Sauerwald, T.; Reimringer, W.; Ventura, G.; and Schütze, A.: Selective detection of hazardous VOCs for indoor air quality applications using a virtual gas sensor array, J. Sens. Sens. Syst., 3, pp. 253-263; doi: 10.5194/jsss-3-253-2014, 2014.

[8] Baur, T.; Schütze, A.; and Sauerwald, T.: „Optimierung des temperaturzyklischen Betriebs von Halbleitergassensoren", tm-Technisches Messen 82 (4), doi:10.1515/teme-2014-0007, 2015.

[9] DIN EN 50194-1, Elektrische Geräte für die Detektion von brennbaren Gasen in Wohnhäusern, German industry standard, 2000.

[10] Backhaus, K.; Erichson, B.; Plinke, W., and Weiber, R.: „Multivariate Analysemethoden“ Springer-Verlag, ISBN 3540-67146-3, 2000. 